Case Report

\title{
A Case of Small Bowel Obstruction and Enterocutaneous Fistulation Resulting from a Mesenteric Haematoma following Blunt Abdominal Trauma
}

\author{
Matthew North, Nicholas Aveyard, Oyeniyi Diya, \\ Jeremy Berger, and Maitham Al-Whouhayb \\ Department of General Surgery, Barnet and Chase Farm Hospitals, Royal Free London NHS Foundation Trust, London, UK \\ Correspondence should be addressed to Maitham Al-Whouhayb; maitham.alwhouhayb@nhs.net
}

Received 27 June 2017; Revised 11 September 2017; Accepted 18 September 2017; Published 26 November 2017

Academic Editor: Oded Olsha

Copyright (C) 2017 Matthew North et al. This is an open access article distributed under the Creative Commons Attribution License, which permits unrestricted use, distribution, and reproduction in any medium, provided the original work is properly cited.

A 23-year-old male with a history of previous abdominal surgery was involved in a road traffic accident. He was discharged after initial assessment but represented several days with small bowel obstruction secondary to a mesenteric haematoma. He underwent resection and recovered well but represented later on the day of discharge with a leaking surgical wound consistent with an enterocutaneous fistula. This was managed conservatively and closed spontaneously after ten days. This case serves to highlight that adhesions from previous surgery can tether the small bowel causing mesenteric injury following blunt-force trauma. It also demonstrates that postoperative ileus can result in an enterocutaneous fistula that has the appearance of an anastomotic breakdown but which resolves more rapidly.

\section{Case Report}

A 23-year-old male was taken to a general hospital after the car he was travelling in collided with a tree at fifty miles per hour. CT demonstrated free fluid in the pelvis, likely from a mesenteric bleed, but no free air and no solid organ damage (Figure 1). He was transferred to a major trauma centre where he was managed conservatively and discharged the next day.

Five days later he represented to a third hospital with severe abdominal pain. A repeat CT showed an increase in the volume of pelvic free fluid and he was immediately taken to theatre for exploratory laparotomy. This revealed a midsmall bowel point of obstruction caused by adhesion to a mesenteric haematoma. Extensive adhesions were evident (the patient had an appendicectomy age nine), but there was no prior history of symptoms of obstruction. Proximal adhesiolysis was performed to allow mobilisation of bowel but this was limited by extensive peritoneal contamination and patient stability; distal and pelvic adhesions therefore remained.

Although there was no full thickness or serosal injuries to the small bowel, it was completely detached from the mesentery and showed signs of ischaemia. A $20 \mathrm{~cm}$ section of small bowel was resected, with a side-by-side anastomosis fashioned. Histology from the resected section of small bowel showed only inflammatory and fibrotic changes compatible with trauma.

After four days in ITU, a repeat CT scan was performed as NG aspirate volumes and inflammatory markers remained high. This showed a small left iliac fossa collection with no clear features of perforation. There was small bowel dilatation with a change of calibre in the region of the anastomosis, raising the possibility of obstruction (Figures 2(a) and 2(b)). This most likely represented a paralytic ileus and it resolved without further intervention. The patient was discharged twelve days after surgery.

However, he represented later that day with a leaking abdominal surgical wound. The fluid had the appearance of undigested food, raising the possibility of an enterocutaneous fistula (ECF). A further CT scan revealed two suspected collections in the lower abdomen and pelvis, with one of these communicating with small bowel at the anastomosis and fistulating through the anterior abdominal wall. After 


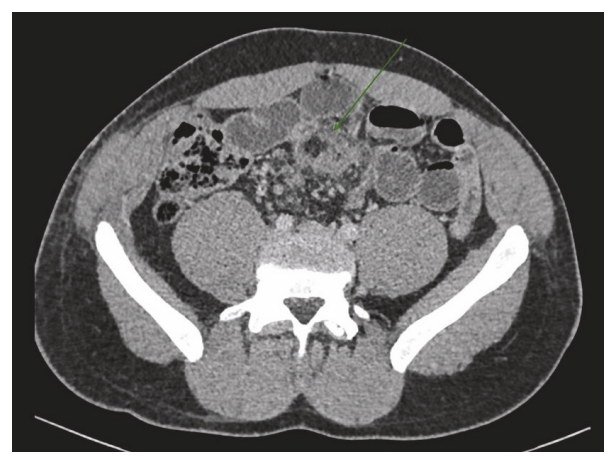

FIgURE 1: Contrast enhanced axial CT image at the time of injury demonstrated high attenuation haematoma within the small bowel mesentery (arrow).

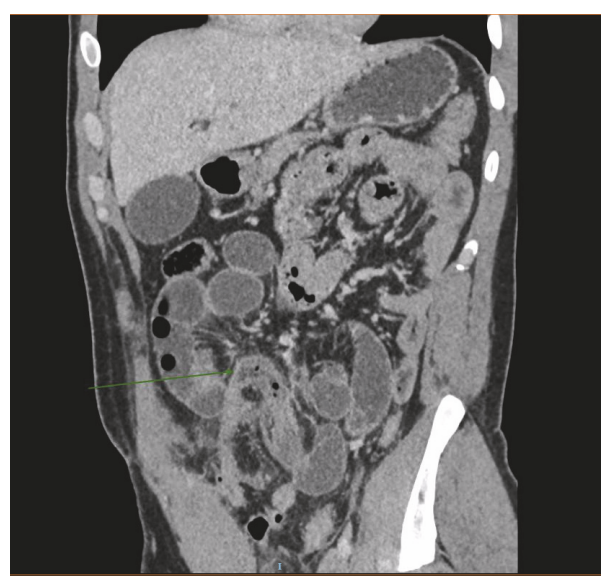

(a)

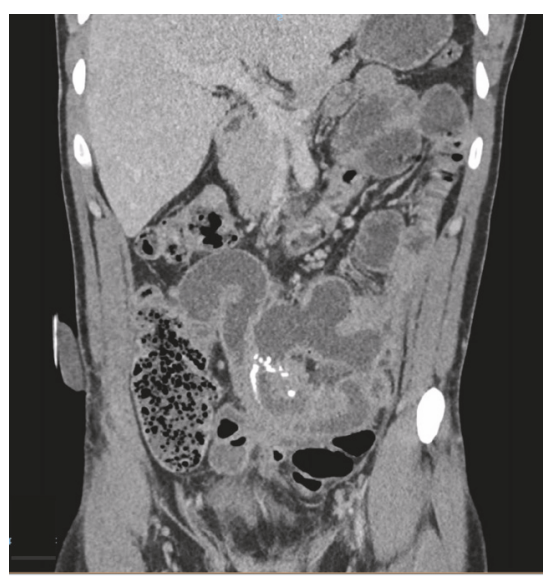

(b)

FIGURE 2: ((a) and (b)) Contrast enhanced coronal CT images 5 days after trauma: there is small bowel dilatation with an acute calibre change of the small bowel indicating obstruction at the position of the previous mesenteric haematoma (arrow in (a)).

seven days of IV antibiotics, the wound output reduced to nothing and his inflammatory markers resolved.

The patient was reviewed in the outpatient clinic five weeks later. The surgical wound had healed well, with no discharge and no signs of herniation.

\section{Discussion}

Mesenteric haematomas following blunt abdominal trauma are a recognised but rare cause of bowel obstruction and perforation (reviewed in [1]). In blunt abdominal trauma (BAT), such as that encountered in a road traffic accident, the abdominal organs most commonly involved are the liver and spleen [2]. These structures are relatively immobile, making them vulnerable to rapid acceleration and deceleration forces.

However, small bowel injury is also common in BAT [3]. In a large study at a major trauma centre, $13 \%$ of total admissions requiring laparotomy over a 5 -year period were due to BAT. Of these, $13 \%$ of patients suffered mesenteric injuries [4]. Other studies have detected bowel and mesenteric injuries in $5 \%$ of BAT at laparotomy [5]. Several mechanisms of injury have been proposed, including the "fixed point" theory, whereby tangential tears occur at relatively fixed points along the bowel [3]. This theory has been supported by an historic study showing that the majority of small bowel injuries resulting from trauma occur in the proximal jejunum or distal ileum [6]. Injuries in these regions are more likely to be mesenteric damage rather than primary bowel perforation [3].

In the case described here, adhesions from the prior appendicectomy acted to tether the mid-small bowel, making it susceptible to deceleration injury and causing a mesenteric bleed. The resulting haematoma subsequently resulted in obstruction.

Diagnosis of bowel and mesenteric injuries can be difficult, and initial CT scans are often nonspecific [5]. Even short delays in diagnosis of hollow viscus injury in BAT have been shown to increase mortality [7]. Although there are few data on the proportion of "missed" injuries with delayed diagnosis, one multicentre study showed that $27 \%$ of patients had a delay in operative intervention, with a corresponding increase in mortality [8]. In the absence of specific CT findings, correlation of nonspecific CT features with clinical findings is recommended, with repeat CT scanning 6-8 hours following patient stabilisation [5]. 
Management of free fluid found alone on CT without organ injury is controversial, as improving imaging technology has led to an increase in conservative treatment. Several studies have attempted to provide clarity (reviewed in [9]). Banz et al. have proposed a treatment algorithm based on five simple questions, including an assessment of free fluid location and volume, as well as the clinical status of the patient [9]. Cases such as this one, where there is free fluid but no obvious organ injury, are the most difficult to manage, especially in a younger patient with a large haemodynamic reserve. Patients with a significant mechanism of injury (e.g., road traffic accident) are at high risk of a missed visceral injury and should be closely observed in the early postinjury period.

Enterocutaneous fistulae can result from a number of intra-abdominal insults, including inflammatory bowel disease and elective surgery. Trauma, however, is considered a rare cause of ECF [10]. A small-scale study has shown that nearly one-third of posttraumatic ECF heal spontaneously, without the need for further operative intervention [10]. A larger ten-year study of ECF, from a single major trauma centre, determined that $74 \%$ of ECF occurred in patients with temporary abdominal closure and were more likely to arise from the small bowel [10] (as in this case). $40 \%$ of ECF resulted from anastomotic breakdown, with an average time to spontaneous closure in this study of 34 days [11]. The rapid closure of the ECF described here is unusual and is likely due to the distal obstruction postoperatively (ileus) causing backpressure through the anastomosis, and so creating a fistula, rather than anastomotic breakdown. As the ileus resolved, this pressure dropped and was no longer sufficient to keep the fistula open, causing rapid closure.

This case highlights a rare but significant cause of small bowel obstruction following trauma, which can be delayed and easily overlooked in the context of polytrauma [12] and in the absence of serial cross-sectional imaging [5].

\section{Consent}

Written consent has been obtained from the patient.

\section{Conflicts of Interest}

The authors declare that they have no conflicts of interest.

\section{References}

[1] A. H. Jahromi, L. Johnson, and A. M. Youssef, "Delayed small bowel perforation following blunt abdominal trauma: A case report and review of the literature," Asian Journal of Surgery, vol. 39, no. 2, pp. 109-112, 2016.

[2] R. K. Pokharna and V. Shanker, "Small bowel stricture after blunt abdominal trauma: missed diagnosis?" Tropical gastroenterology : official journal of the Digestive Diseases Foundation, vol. 32, no. 4, pp. 256-258, 2011.

[3] A. H. Dauterive, L. Flancbaum, and E. F. Cox, "Blunt intestinal trauma. A modern-day review," Annals of Surgery, vol. 201, no. 2, pp. 198-203, 1985.
[4] E. F. Cox, "Blunt abdominal trauma. A 5-year analysis of 870 patients requiring celiotomy," Annals of Surgery, vol. 199, no. 4, pp. 467-474, 1984.

[5] N. Brofman, M. Atri, J. M. Hanson, L. Grinblat, T. Chughtai, and F. Brenneman, "Evaluation of bowel and mesenteric blunt trauma with multi-detector CT," RadioGraphics, vol. 26, no. 4, pp. 1119-1131, 2006.

[6] V. S. Counsellor and C. J. McCormack, "Subcutaneous perforation of the jejunum," Annals of Surgery, vol. 102, pp. 365-374, 1935.

[7] D. J. Malinoski, M. S. Patel, D. O. Yakar et al., "A diagnostic delay of 5 hours increases the risk of death after blunt hollow viscus injury," Journal of Trauma - Injury Infection and Critical Care, vol. 69, no. 1, pp. 84-87, 2010.

[8] S. M. Fakhry, M. Brownstein, D. D. Watts, C. C. Baker, and D. Oller, "Relatively short diagnostic delays ( $<8$ hours) produce morbidity and mortality in blunt small bowel injury: An analysis of time to operative intervention in 198 patients from a multicenter experience," Journal of Trauma - Injury Infection and Critical Care, vol. 48, no. 3, pp. 408-415, 2000.

[9] V. M. Banz, M. U. Butt, H. Zimmermann, V. Jeger, and A. K. Exadaktylos, "Free abdominal fluid without obvious solid organ injury upon CT imaging: an actual problem or simply overdiagnosing?" Journal of Trauma Management \& Outcomes, vol. 3, no. 1, 2009.

[10] K. J. Buechter, D. Leonovicz, P. R. Hastings, and C. Fonts, "Enterocutaneous fistulas following laparotomy for trauma," The American Surgeon, vol. 57, no. 6, pp. 354-358, 1991.

[11] P. E. Fischer, T. C. Fabian, L. J. Magnotti et al., "A ten-year review of enterocutaneous fistulas after laparotomy for trauma," The Journal of Trauma: Injury, Infection, and Critical Care, vol. 66, no. 4, pp. 1052-1058, 2009.

[12] J. C. Allen, "Post-traumatic small bowel obstruction.," Journal of the Royal Army Medical Corps, vol. 140, no. 1, pp. 47-48, 1994. 


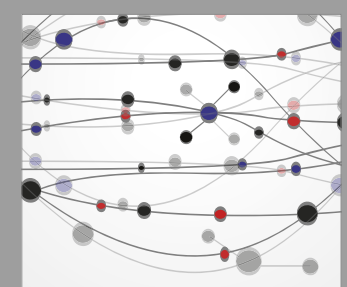

The Scientific World Journal
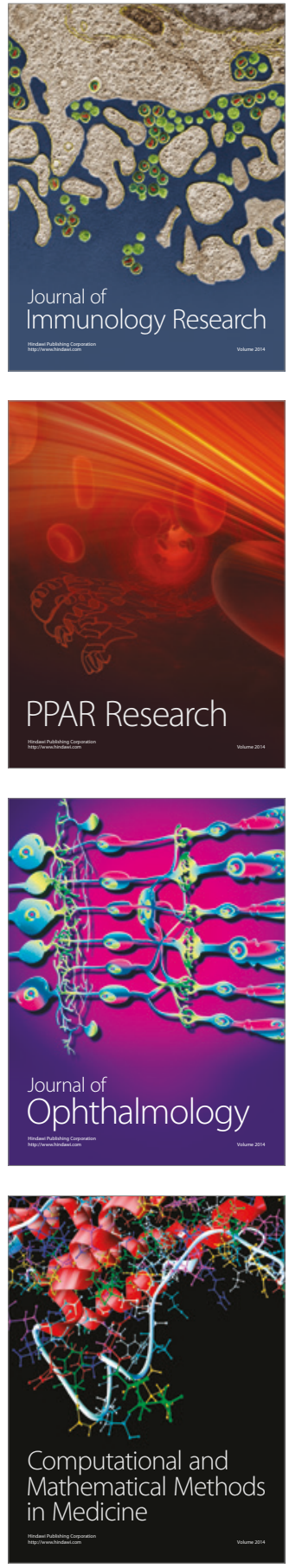

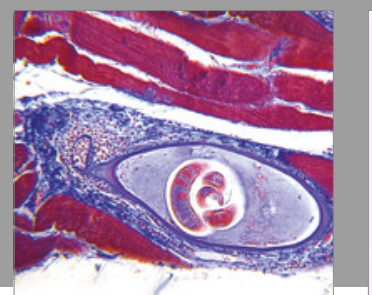

Gastroenterology Research and Practice
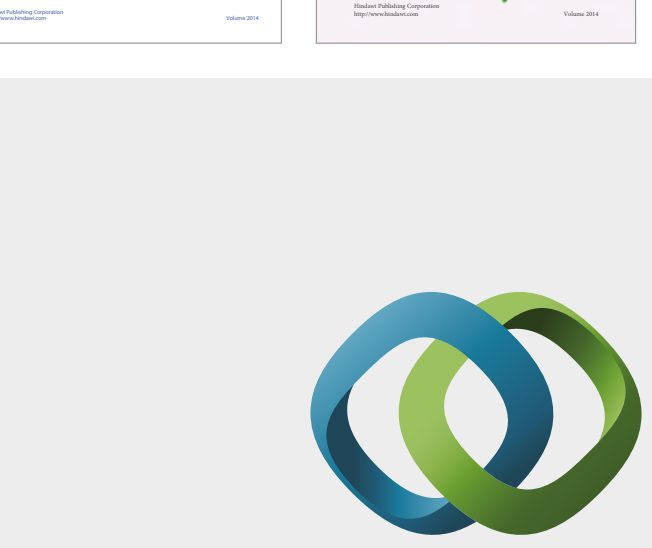

\section{Hindawi}

Submit your manuscripts at

https://www.hindawi.com
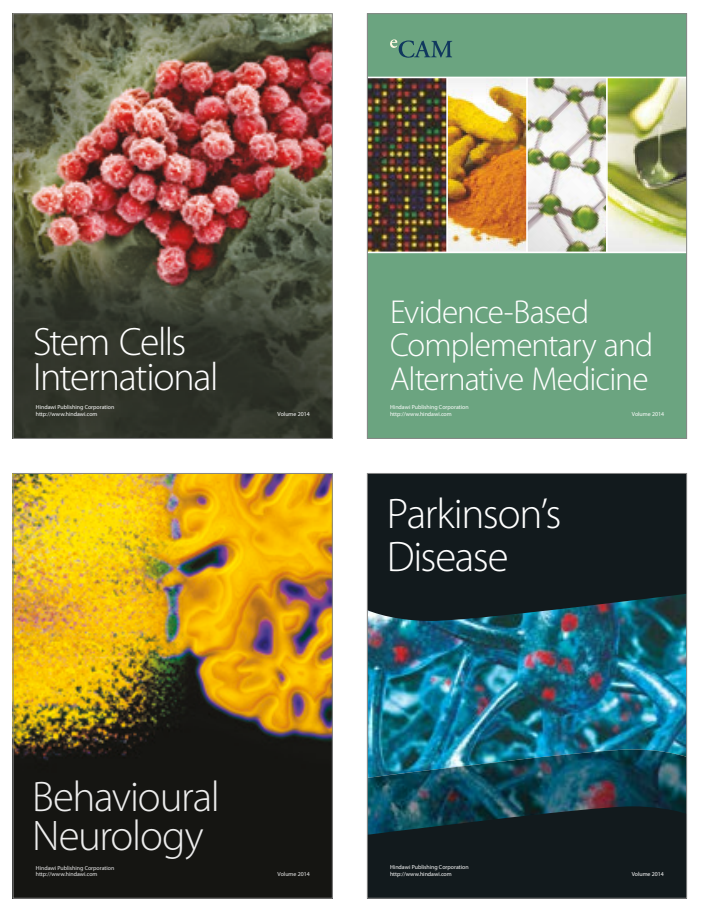
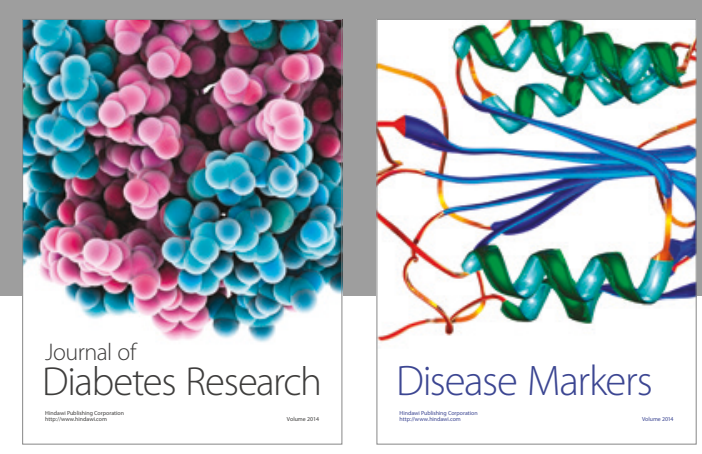

Disease Markers
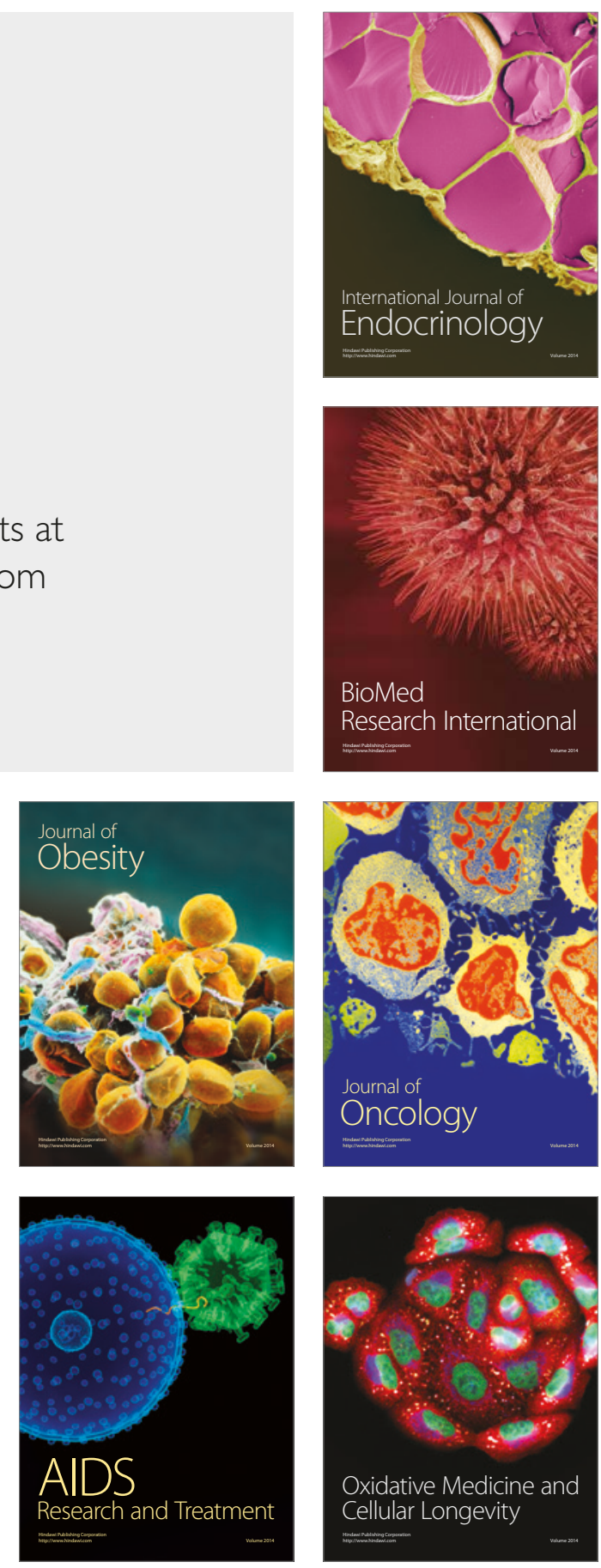